\title{
Effect of relaxation on self-induced transparency
}

\author{
Munir H. Nayfeh \\ Physics Department, Yale University, New Haven, Connecticut 06520
}

Ali H. Nayfeh

Virginia Polytechnic Institute and State University, Blacksburg, Virginia 24060

(Received 11 July 1977)

\begin{abstract}
We derive an equation of motion of the Bloch-vector tipping angle and the area theorem in the presence of relaxation. We derive relaxation-dependent shifts in the equilibrium $n \pi$ areas. Part of these shifts depends on a weighted time integral of the sine of the area, and hence these shifts remove the propagation degeneracy of pulses having equal areas but different shapes.
\end{abstract}

In self-induced transparency ${ }^{1}$ (SIT) studies, special attempts were taken to minimize the probability of SIT being confused with incoherent saturation, since the nonlinear transmission in SIT could be interpreted as a "hole-burning" or bleaching effect, where the absorption profile is completely saturated by the leading edge of the pulse. ${ }^{2}$ In fact a clear-cut demonstration of SIT was not achieved until gaseous absorbers were investigated. ${ }^{3}$ Specific studies of phase relaxation of propagation were carried out experimentally and theoretically..$^{1-9}$ The effect of a phase relaxation time $T_{2}$ on SIT was investigated by warming a ruby sample and studying the transmission in it as a function of temperature. ${ }^{4}$ Theoretically, a computer simulation of the ruby SIT experiment of Ref. 4 was carried out, where the value of the phase-memory time is the order of the pulse length. The numerical and experimental results ${ }^{4}$ showed that the transmission and pulse delay are reduced particularly for near $\pi$ pulses. The reduction is considerably less for pulses with areas greater than $2 \pi$. Also, a computer simulation of the mercury SIT experiment was carried out with pulse widths as large as $10 \%$ of the relaxation widths. ${ }^{3}$

Here we study the effect of relaxation on SIT by deriving an equation for the Bloch-vector tipping angle and the area theorem when the relaxation widths are not negligible in comparison with the duration of the pulses. The limit where the pulse duration is much shorter than the relaxation times was treated previously.,2 In this limit an atom undergoes practically no damping during its short interaction with the pulse, and the system can be described by the undamped Bloch equations. Higher corrections, the order of $\gamma_{1}$ and $\gamma_{2}$, to the undamped system were given analytically by McCall and Hahn. ${ }^{1}$ On the other hand, the rate-equation time limit of the interaction was developed when the relaxation is very large, and a solution was presented in terms of a time-vary- ing transmittance. ${ }^{7}$ The present work extends these studies by deriving the equation of motion of the Bloch-vector tipping angle without assuming small damping. This equation includes the sum of the infinite-order corrections in the relaxation widths. Moreover, in the present treatment, we derive an area theorem in the presence of relaxation. The relaxation causes shifts in the equilibrium $n \pi$ areas, which depend on the relaxation-weighted time-integrated sine of the area. These shifts remove the propagation degeneracy of pulses having equal areas but different shapes. Also, the present study shows as expected that pulses having larger areas are less affected by relaxation than pulses having smaller areas and comparable duration. With damping, the optical Bloch equations are ${ }^{2}$

$$
\begin{aligned}
& \frac{d u}{d t}+\gamma_{2} u=-\Delta v, \\
& \frac{d v}{d t}+\gamma_{2} v=\Delta u+\mu \mathcal{E} w, \\
& \frac{d w}{d t}+\gamma_{1} w=-\mu \mathcal{E} v-\gamma_{1},
\end{aligned}
$$

where $\Delta=\omega-\omega_{0}$ is the detuning, $\omega_{0}$ is the transition frequency, $\mathcal{E}(t)$ is the amplitude of the electric field $E$ defined by $E=\mathcal{E}(t) \cos \omega t, u$ and $v$ are the dipole dispersive and absorptive components, $w$ is the inversion, $\mu$ is the dipole moment in units of $\hbar$, and $\gamma_{1}=T_{1}^{-1}$ and $\gamma_{2}=T_{2}^{-1}$ are the atomic-energy and phase-relaxation widths, respectively.

To solve Eqs. (1)-(3), we first introduce new variables defined by $u=\tilde{u} \exp \left(-\gamma_{2} t\right), v=\tilde{v} \exp \left(-\gamma_{2} t\right)$, and $w=\tilde{w} \exp \left(-\gamma_{2} t\right)$. Then, Eqs. (1)-(3) become

$$
\begin{aligned}
& \frac{d \tilde{u}}{d t}=-\Delta \tilde{v}, \\
& \frac{d \tilde{v}}{d t}=\Delta \tilde{u}+\mu \mathcal{E} \tilde{w}, \\
& \frac{d \tilde{w}}{d t}=\mu \mathcal{E} \tilde{v}-\gamma_{1} e^{\gamma_{2} t}+\left(\gamma_{2}-\gamma_{1}\right) \tilde{w} .
\end{aligned}
$$


When $\Delta=0$ (i.e., the field is exactly tuned), Eq. (4) reduces to $d \tilde{u} / d t=0$. The most common initial condition, in which all atoms are in their ground states, leads to $\tilde{u}(t, \Delta=0)=0$ for all time. The on-resonance equations for $\tilde{v}$ and $\tilde{w}$ can be easily rewritten in terms of the Bloch-vector tipping angle $\theta(t)=\mu \int_{-\infty}^{t} \mathcal{E}(t) d t$ as

$$
\begin{aligned}
& \frac{d \tilde{v}}{d \theta}=\tilde{w}, \\
& \frac{d \tilde{w}}{d \theta}=-\tilde{v}-\frac{\gamma_{1} e^{\gamma_{2}}{ }^{t}}{\mu \mathcal{E}}+\frac{\left(\gamma_{2}-\gamma_{1}\right) \tilde{w}}{\mu \mathcal{E}} .
\end{aligned}
$$

We solve Eqs. (7) and (8) for the case where $\gamma_{2}-\gamma_{1}$ is small compared with $\gamma_{1}$ and $\gamma_{2}$ and with no restriction on the magnitudes of $\gamma_{1}$ and $\gamma_{2}$. To first order, we neglect the term proportional to $\left(\gamma_{2}-\gamma_{2}\right)$, use the method of variation of parameters, and obtain

$$
\begin{aligned}
& \tilde{v}=\tilde{v}_{0}=-\sin \theta-\gamma_{1} \int_{-\infty}^{t} e^{\gamma_{2} t^{\prime}} \sin \left(\theta-\theta^{\prime}\right) d t^{\prime}, \\
& \tilde{w}=\tilde{w}_{0}=-\cos \theta-\gamma_{1} \int_{-\infty}^{t} e^{\gamma_{2} t^{\prime}} \cos \left(\theta-\theta^{\prime}\right) d t^{\prime} .
\end{aligned}
$$

To obtain a correction to Eqs. (9) and (10), we substitute for $\tilde{w}$ from Eq. (10) into Eq. (8) and obtain

$$
\begin{gathered}
\tilde{v}=-\sin \theta-\int_{-\infty}^{t}\left[\gamma_{1} e^{\gamma_{2} t^{\prime}}-\left(\gamma_{2}-\gamma_{1}\right) \tilde{w}_{0}\right] \\
\times \sin \left(\theta-\theta^{\prime}\right) d t^{\prime} .
\end{gathered}
$$

Let us compare the present solution with the perturbative results of McCall and Hahn. For small relaxation, $\tilde{w}_{0}$ can be replaced by $-\cos \theta$ and the exponential can be replaced by 1 in Eq. (11). The result is

$$
\begin{gathered}
\tilde{v}=-\sin \theta-\int_{-\infty}^{t}\left[\gamma_{1}+\left(\gamma_{2}-\gamma_{1}\right) \cos \left(\theta^{\prime}\right)\right] \\
\times \sin \left(\theta-\theta^{\prime}\right) d t^{\prime} .
\end{gathered}
$$

Integrating Eq. (12) by parts and taking the lowerlimit zero in evaluating the integrated terms gives

$$
\begin{aligned}
\tilde{v}= & -\sin \theta-\gamma_{1} \int_{-\infty}^{t} \mu \mathcal{E}\left(t^{\prime}\right) t^{\prime} \cos \left(\theta-\theta^{\prime}\right) d t^{\prime} \\
& +\left(\gamma_{2}-\gamma_{1}\right) \int_{-\infty}^{t} \mu \mathcal{E}\left(t^{\prime}\right) t^{\prime} \cos \left(\theta-2 \theta^{\prime}\right) d t^{\prime},
\end{aligned}
$$

in agreement with the solutions of $\mathrm{McC}$ all and Hahn. ${ }^{1}$

It may be reasonable to assume that the offresonance dipoles respond to the field in the same way as the resonant dipoles but perhaps with a detuning-dependent reduction in amplitude. ${ }^{209}$ This factorization assumption leads in the absence of relaxation to analytical solutions of the coupled
Maxwell-Schrödinger equations which describe the interaction of light with two-level resonant atoms in a nonlinear absorber. More recently, new attempts ${ }^{10}$ were taken to generate solutions of the ideal system without the factorization assumption using a perturbation scheme in the parameter $\Delta / \omega$. Analyticity was only achieved up to $O[\Delta / \omega]^{2}$ and up to this order the absorption components factorize. Here, we use the factorization assumption and write $\tilde{v}(t ; \Delta)=\tilde{v}(t ; 0) F(\Delta)$, where $F(\Delta)$ is the dipole spectral response function. Then it follows from Eq. (6) and the condition $\tilde{w}(-\infty)=-1$ that .

$$
\begin{aligned}
\tilde{w}=-F(\Delta)\left(\cos \theta+\int_{-\infty}^{t}\left[\gamma_{1} e^{\gamma_{2} t^{\prime}}-\left(\gamma_{2}-\gamma_{1}\right) \tilde{w}_{0}\right]\right. \\
\left.\times \cos \left(\theta-\theta^{\prime}\right) d t^{\prime}\right) \\
+[-1+F(\Delta)] f(t),
\end{aligned}
$$

where

$$
f(t)=\frac{\gamma_{1}}{\gamma_{2}} e^{\gamma_{2}}{ }^{t}-\left(\gamma_{2}-\gamma_{1}\right) \int_{-\infty}^{t} \tilde{w}_{0} d t^{\prime}+1-\frac{\gamma_{1}}{\gamma_{2}} .
$$

We can now derive an equation for the Blochvector tipping angle by eliminating $\tilde{u}$ from Eqs. (4) and (5). The result is

$$
\ddot{\theta}+\frac{\dot{f}}{f} \dot{\theta}=-\frac{\Delta^{2}}{[1-F(\Delta)] f(t)} \tilde{v}(t ; \Delta)
$$

or

$$
\begin{aligned}
\ddot{\theta}+\frac{\dot{f}}{f} \dot{\theta}=\frac{1}{\tau^{2} f}\left(\sin \theta+\int_{-\infty}^{t}\right. & {\left[\gamma_{1} e^{\gamma_{2} t^{\prime}}-\left(\gamma_{2}-\gamma_{1}\right) \tilde{w}_{0}\right] } \\
& \left.\times \sin \left(\theta-\theta^{\prime}\right) d t^{\prime}\right),
\end{aligned}
$$

where $\tau^{-2}=\Delta^{2} F(\Delta) /[1-F(\Delta)]$ is the pulse width. We first note that in the absence of relaxation (i.e., $\gamma_{1}=\gamma_{2}=0$ ), Eq. (17) reduces to the previously derived pendulum equation $\ddot{\theta}=\tau^{-2} \sin \theta$. Since $\mathcal{E}$ and $\dot{E}$ vanish at $t= \pm \infty$, the pendulum equation implies a nonoscillatory pendulum which is balanced vertically in the infinite past and swings $360^{\circ}$ to end up balanced in the infinite future. This solution corresponds to the well-known SIT hyperbolic secant pulse.

The presence of relaxation modifies the pendulum equation radically. It introduces time integrals, which make the equation of motion a differential-integral equation. One of the integral contributions is excited by the relaxation width $\gamma_{1}$, while the other contribution is excited by the difference $\gamma_{2}-\gamma_{1}$. We now discuss qualitatively the relaxation effects.

The first contribution is $\dot{f} \dot{\theta} / f$, which introduces damping into the equation of motion. The second 
contribution is a relaxation-dependent restoring force $\tau^{-2} f^{-1}\left(\gamma_{1}, \gamma_{2}, t\right) \sin \theta$. The third contribution is proportional to the energy-changing relaxation width $\gamma_{1}$, and it involves a time integrated $\sin \left(\theta-\theta^{\prime}\right)$ weighted with a relaxation-dependent function. The time-integrated contribution is an accumulative effect which introduces dependence on the details of the pulse shape. This term removes the response degeneracy of pulses having equal areas but different shapes. Note that Eq. (17) ceases to be a pendulum equation as predicted in the ideal systems where relaxation vanishes. The last contribution is proportional to $\gamma_{2}-\gamma_{1}$ and thus vanishes in the case of equal decay widths. Since this term comes from a term which is proportional to $\tilde{w}_{0}$, the angular distribution of this term is different from the third contribution. In the limit of small relaxation, this term leads to the second integral contribution in Eq. (14) whose integrand depends on $\cos \left(\theta-2 \theta^{\prime}\right)$.

Let us consider the importance of the integral contribution excited by the relaxation of the system as a function of the area of the pulse. Let us take $\gamma_{1}=\gamma_{2}=\gamma$, for simplicity. Integrating by parts twice the integral in Eq. (17) and assuming that $\mathcal{E}$ is small, we obtain

$$
\begin{aligned}
\ddot{\theta}+\gamma \dot{\theta}=\frac{\bar{e}^{\gamma t}}{\tau^{2}} & \left(\sin \theta+\frac{\gamma}{\mu^{2} \mathcal{E}^{2}+\gamma^{2}}\right. \\
& \left.\times\left[-\gamma \sin \theta-\mu \mathcal{E} \cos \theta+\mu \mathcal{E} e^{\gamma t}\right]\right) .
\end{aligned}
$$

Equation (18) shows that the integral contribution is smaller for pulses having large area $(\mu \mathcal{E}$ is large) than that for pulses having smaller areas and comparable widths. This result is physically equivalent to the following statement: in the propagation of equal area pulses, pulses of shorter widths are least affected by relaxation.

In order to study propagation effects, we consider the in-quadrature Maxwell equation in the presence of damping; that is,

$$
\begin{aligned}
2\left(K \frac{\partial}{\partial z}+\frac{1}{2} \Gamma k c\right. & \left.+k \frac{\partial}{\partial c t}\right) \mathcal{E}(t, z) \\
& =2 \pi k^{2} N d \int v\left(t, z ; \Delta^{\prime}\right) g\left(\Delta^{\prime}\right) d \Delta^{\prime},
\end{aligned}
$$

where

$$
g\left(\Delta^{\prime}\right)=\frac{\omega_{I}}{\pi} \frac{1}{\left(\Delta^{\prime}-\Delta\right)^{2}+\omega_{I}^{2}}
$$

is an inhomogeneous line-shape detuning function defined as the fraction of dipoles within the detuning interval $d \Delta^{\prime}$ and $\omega_{I}$ is the inhomogeneous half width at half maximum of $g\left(\Delta^{\prime}\right)$. Substituting for $v(t, \Delta)$ into $\mathrm{Eq} .(19)$ yields

$\left(K \frac{\partial}{\partial z}+\frac{1}{2} \Gamma k c+\frac{k}{c} \frac{\partial}{\partial t}\right) E=-\pi k^{2} N d e^{-\gamma_{2} t}\left(\int F\left(\Delta^{\prime}\right) g\left(\Delta^{\prime}\right) d \Delta^{\prime}\right)\left(\sin \theta+\int_{-\infty}^{t}\left[\gamma_{1} e^{\gamma_{2} t^{\prime}}-\left(\gamma_{2}-\gamma_{1}\right) \tilde{w}_{0}\right] \sin \left(\theta-\theta^{\prime}\right) d t^{\prime}\right)$.

Defining a local-time coordinate $\xi=t-z / V$, where $V$ is the constant-pulse velocity, we rewrite Eq. (21) as

$$
\begin{aligned}
\frac{d E}{d \xi}+\hat{\Gamma} E=\frac{\mu}{\chi^{2}} e^{-\gamma_{2} t}\left(\sin \theta+\int_{-\infty}^{t}\right. & {\left[\gamma_{1} e^{\gamma_{2} t^{\prime}}-\left(\gamma_{2}-\gamma_{1}\right) \tilde{w}_{0}\right] } \\
& \left.\times \sin \left(\theta-\theta^{\prime}\right) d t^{\prime}\right),
\end{aligned}
$$

where

$$
\begin{aligned}
& \hat{\Gamma}=-\frac{\Gamma k c}{2(K / V-k / c)}, \\
& \frac{1}{\chi^{2}}=\frac{\pi k^{2} N d}{\mu(K / V-k / c)} \int F\left(\Delta^{\prime}\right) g\left(\Delta^{\prime}\right) d \Delta^{\prime} .
\end{aligned}
$$

Comparing Eqs. (17) and (22), we find that they are equivalent provided that $\chi^{2}=\mu \tau^{2} f e^{-\gamma} 2^{t}$ and $\dot{f} / f=\hat{\Gamma}$.

Because of the presence of a damping time
$T=\gamma^{-1}$, which may be longer than the observation time $\bar{t}-t_{0}$, the McCall-Hahn area theorem derived in what is called the "modern" case will not be applicable. In the "modern" case, one considers free-oscillation times $\bar{t}-t_{0}$ so short $\left.\left[\bar{t}-t_{0}\right) \ll T\right]$ that none of the dipole transients will be damped out. However, because of the dipole dephasing due to inhomogeneous broadenings, the macroscopic polarization will be completely damped out if the observation time is much longer than the inhomogeneous lifetime $T^{*}$. The inhomogeneous lifetime $T^{*}$ is implied by the finite width of $g\left(\Delta^{\prime}\right)$, Eq. (20), and defined in terms of its maximum: $T^{*}=\pi g\left(\Delta^{\prime}\right)_{\max }=\omega_{1}^{-1}$. Some progress can be achieved analytically in the case of moderate damping times, that is, damping times that are comparable with the observation time. In this case, we use a perturbation scheme in deriving the area theorem. To first order, the "modern" case is 
assumed to apply. Therefore, integrating Eq. (19) up to times large enough for the pulse to die out, one obtains

$$
\frac{d A}{d z}\left(t_{0}, z\right)+\bar{\Gamma} A\left(t_{0}, z\right)=\alpha v\left(t_{0}, z ; 0\right),
$$

where $t_{0}$ is a time when the pulse has died out; $\alpha$ $=\left(\pi^{2} N \hbar \mu^{2} k^{2} / K\right) g(0)$ and $A(t, z)$ and $\bar{\Gamma}$ replace $\theta(t, z)$ and $\frac{1}{2} \Gamma k c$, respectively. In the absence of damping $v\left(t_{0}, z ; 0\right)$ is given by $-\sin A\left(t_{0}\right)$. The next approximation in the presence of damping is included by introducing the effect of damping on $v\left(t_{0}, z ; 0\right)$ through a correction to $-\sin A\left(t_{0}\right)$. This correction is given by Eq. (11). Substituting for $v$ into Eq. (24), we obtain

$$
\begin{array}{r}
\frac{d A}{d z}+\bar{\Gamma} A=-\alpha e^{-\gamma_{2} t_{0}}\left(\sin A+\int_{-\infty}^{t_{0}}\left[\gamma_{1} e^{\gamma_{2} t^{\prime}}-\left(\gamma_{2}-\gamma_{1}\right) \tilde{w}_{0}\right]\right. \\
\left.\times \sin \left(A-A^{\prime}\right) d t^{\prime}\right) .
\end{array}
$$

Now we consider the effect of the dissipative feature of the system on the propagation of $n \pi$ pulses. Consider $\gamma_{1}=\gamma_{2}=\gamma$. When $A\left(t_{0}, z\right)=n \pi$, Eq. (25) becomes

$$
\frac{d A}{d z}+\bar{\Gamma} A=(-1)^{n} \alpha e^{-\gamma t_{0}}\left(\gamma \int_{-\infty}^{t} e^{\gamma t^{\prime}} \sin A^{\prime} d t^{\prime}\right),
$$

which indicates that $n \pi$ areas are no longer equili- . brium areas due to relaxation. The relaxation destroys that in two ways. The first one is through a simple exponential decay of the area due to absorption or scattering of the pulse as it travels through the medium. This process is described by the term $\bar{\Gamma} A$ in Eq. (26): Relaxation affects $n \pi$ pulses in a more detailed way too. This detailed dependence is aperiodic since it involves sine of the area, and therefore the sign of the effect depends on $n$ as shown in Eq. (26). Note that it also depends on the previous history of the pulse through its dependence on the time-integrated area. Since $d t^{\prime}$ can be written as $d A^{\prime} / \mu \mathcal{E}$, the time-integrated area is then weighted by the field amplitude and the relaxation evolution $\exp (\gamma t)$. This of course removes the degeneracy of pulses having equal areas but having different time distributions.

When $\theta \ll \pi$ Eq. (19) reduces to the linear system

$$
\begin{aligned}
2\left(K \frac{\partial}{\partial z}+\bar{\Gamma}+K \frac{\partial}{\partial c t}\right) & \mu E \\
= & -\left(2 \pi \mu k^{2} N d \int_{-\infty}^{\infty} F\left(\Delta^{\prime}\right) g\left(\Delta^{\prime}\right) d \Delta^{\prime}\right) \\
& \times \int_{-\infty}^{t} e^{\gamma\left(t^{\prime}-t\right)} \mu E\left(t^{\prime}\right) d t^{\prime}
\end{aligned}
$$

Equation (27) is consistent with linearizing the system (1)-(3) in the weak-field case by substituting -1 for $w$ in Eq. (2). This result, Eq. (27) when $\bar{\Gamma}$ is neglected, agrees with the study carried out by Crisp ${ }^{11}$ on propagation of small areas.

In the absence of relaxation, $A=A_{0}=4 \tan ^{-1}$ $\times[\exp (\xi / \tau)]$ and the equilibrium areas are $n \pi$, where $n=1,2,3, \ldots$ The areas corresponding to even values of $n$ are stable while those corresponding to odd values of $n$ are unstable. For small $\gamma, A=4 \tan ^{-1}[\exp (\xi / \tau)]+\delta A$ and the equilibrium areas are given by $n \pi+e$, where $\delta A$ and $e$ are small. It follows from Eq. (25) that

$$
e=(-1)^{n+1} \bar{\Gamma} n \pi \alpha^{-1} e^{\gamma t_{0}}+\gamma I,
$$

where

$$
\begin{aligned}
I & =\int_{-\infty}^{t_{0}} e^{\gamma t^{\prime}} \sin A_{0}^{\prime} d t^{\prime} \\
& =4 \tau \int_{0}^{e^{t_{0} / \tau}} Z^{\tau \gamma}\left(1-Z^{2}\right)\left(1+Z^{2}\right)^{-2} d Z .
\end{aligned}
$$

The sign of the first term in Eq. (28) depends on $n$, while its magnitude is independent of the pulse shape. Thus, this term tends to increase equilibrium areas (corresponding to even values of $n$ ). The second shift in the equilibrium location $\gamma I$ is only a function of $\tau \gamma$ and $t_{0} / \tau$ and it is independent of $n$. Moreover, it is always positive and hence tends to increase the location of the equilibrium points.

Let us now examine the effect of relaxation on the propagation of general area pulses. Integrating by parts twice the integral in Eq. (25) when $\gamma_{1}=\gamma_{2}$ $=\gamma$, and neglecting $\dot{\mathcal{E}}$, we obtain

$$
\begin{aligned}
\frac{d A}{d z}+\bar{\Gamma} A=-\alpha e^{-\gamma t_{0}} & \left(\sin A+\frac{\gamma}{\mu^{2} \mathcal{E}^{2}+\gamma^{2}}\right. \\
& \left.\times\left(-\gamma \sin A+\mu \mathcal{E} \cos A-\mu \mathcal{E} e^{\gamma t_{0}}\right)\right) .
\end{aligned}
$$

Equation (30) shows that, for comparable durations, pulses having large areas (i.e., large $\mu \mathcal{E}$ ) contribute less to the time-integrated term in Eq. (25). Therefore, pulses having large areas are affected less by the relaxation of the system. This conclusion is in agreement with the computer simulation of the mercury experiment where the maximum transmission is least shifted for larger areas. $^{3}$ The experiment also seems to indicate this behavior. ${ }^{3}$ Physically, this effect is equivalent to saying that in the case of equal areas shorter pulses are less affected by relaxation than longer pulses. Moreover, large-area pulses imply stimulated emission and absorption of many photons during the duration of the pulse, and 
therefore for a given $\gamma t_{0}$, one can conclude, as indicated by the above equations, that large-area pulses are affected less by decay than small-area pulses where stimulated emission and absorption are smaller.

In conclusion, we have derived equations of motion for the Bloch-vector tipping angle and the area theorem in the presence of relaxation. These equations involve relaxation-weighted time-integrated sine and cosine of the area of the pulses which remove the propagation degeneracy of pulses having equal areas but different shapes. Shifts in the $n \pi$ equilibrium areas are calculated which depend on the above time-integration effect. It is also found that for comparable durations pulses having large areas are affected less by the relaxation of the system than pulses having small areas.

\section{ACKNOWLEDGMENT}

We are grateful to Professor E. L. Hahn and Professor A. Szöke for their useful discussions.
${ }^{1}$ S. L. McCall and E. L. Hahn, Phys. Rev. Lett. 18, 908 (1967); Phys. Rev. 183, 457 (1969).

${ }^{2} \mathrm{~L}$. Allen and J. H. Eberly, Optical Resonance and TwoLevel Atoms (Wiley-Interscience, New York, 1975), Chap. 5 and references in Chap. 5.

${ }^{3}$ H. M. Gibbs and R. E. Slusher, Phys. Rev. A 6, 2326 (1972); R. E. Slusher and M. H. Gibbs, ibid. $\underline{\overline{5}}, 1634$ (1972); 6, 1255E (1972).

${ }^{4}$ I. M. Asher, Phys. Rev. A 5, 349 (1972); I. M. Asher and M. D. Scully, Opt. Commun. $\underline{3}$, 394 (1971).

${ }^{5}$ F. A. Hopf and M. O. Scully, Phys. Rev. B 1, 50 (1970).

${ }^{6}$ E. Courtens and A. Szöke, Phys. Lett. 28A, 296
(1968).

${ }^{7}$ A. C. Selden, Br. J. Appl. Phys. 18, 743 (1967).

${ }^{8}$ F. Gires and F. Combaud, J. Phys. (Paris) 26, 325 (1965).

${ }^{9}$ L. Matulic and J. H. Eberly, Phys. Rev. A 6, 822, 1258(E) (1972); R. A. Marth, D. A. Holmes, and J. H. Eberly, ibid. 9, 2733 (1974).

${ }^{10} \mathrm{~L}$. Matulic, in Proceedings of the Fourth Rochester Conference on Coherence and Quantum Optics, 8-10 June, 1977 (unpublished).

${ }^{11}$ M. D. Crisp, Phys. Rev. A 1 , 1604 (1970). 